\title{
How Should We Manage Iatrogenic Perforation Caused by Colonoscopy?
}

\author{
Eun Sun Kim \\ Division of Gastroenterology and Hepatology, Department of Internal Medicine, Korea University College of Medicine, Seoul, Korea
}

See "Optimal Methods for the Management of Iatrogenic Colonoscopic Perforation" by Dae Kyu Shin, Sun Young Shin, Chi Young Park, et al., on page 282-288.

Colonoscopy is widely performed in Korea for screening, diagnostic, and therapeutic purposes. However, serious complications of colonoscopy, such as iatrogenic colon perforation, have been reported. The rate of perforation in colonoscopy is $0.1 \%$ to $0.3 \%{ }^{1,2}$ Perforation occurs in $0.19 \%$ to $0.29 \%$ of colon polypectomies, ${ }^{3,4}$ and the risk increases to $1.9 \%$ in elderly patients. ${ }^{5}$ Colon perforation is associated with high morbidity and mortality, and adequate management and decision-making strategy is necessary. ${ }^{4,6}$

How should we manage iatrogenic perforation caused by colonoscopy? Decision-making is important because of the associated unsatisfactory clinical course, prolonged hospital day, increased cost, malpractice claims, and patient dissatisfaction.

In this issue of Clinical Endoscopy, Shin et al. ${ }^{4}$ reported the clinical outcomes of several management strategies for iatrogenic colonoscopic perforations. Twenty of 41 patients underwent conservative management, with a success rate of $90 \%$. Two patients were converted to surgical management after the failure of initial conservative management. Surgical manage-

Received: May 7, 2016 Revised: May 20, 2016

Accepted: May 23, 2016

Correspondence: Eun Sun Kim

Division of Gastroenterology and Hepatology, Department of Internal Medicine, Korea University Anam Hospital, Korea University College of Medicine, 73 Inchon-ro, Seongbuk-gu, Seoul 02841, Korea

Tel: +82-2-920-6555, Fax: +82-2-923-1943, E-mail: silverkes@naver.com

(cc) This is an Open Access article distributed under the terms of the Creative Commons Attribution Non-Commercial License (http://creativecommons.org/ licenses/by-nc/3.0) which permits unrestricted non-commercial use, distribution, and reproduction in any medium, provided the original work is properly cited. ment was performed in 23 patients, including two who failed conservative management. Of 14 patients who underwent surgery at 8 hours after the perforation, there was no significant difference in adverse outcomes between the laparotomy group and the laparoscopic surgery group. The medical costs and claim rate were 1.45 and 1.87 times greater in the exploratory laparotomy group, respectively. Shin et al. ${ }^{4}$ concluded that conservative management of colonoscopic perforation could be an option for patients without overt symptoms of peritonitis or with a small defect size. If surgical management is required, laparoscopic surgery may be considered as the initial procedure even with a delayed diagnosis.

In this study, the number of enrolled patients was relatively small, and the data were collected retrospectively; therefore, prospectively randomized controlled data are needed to establish management strategies with strong evidence. Despite this limitation, we have several management option for iatrogenic colon perforation.

First, we can use endoscopic closure with a clip device in limited situations. The perforation must be found immediately after colonoscopy, the bowel preparation should be clean, the perforation should be smaller than $10 \mathrm{~mm}$, and the patient's condition should be good, with no evidence of peritonitis. Of course, endoscopic clipping should be performed by experienced endoscopists. For a more effective and comfortable procedure, $\mathrm{CO}_{2}$ insufflation is recommended. An overthe-scope device is also recommended for effective closure of perforations at a center with this availability.

Second, laparoscopic surgery can be a choice for manage- 
ment of iatrogenic perforation. This can be performed when medical treatment has failed, without severe peritonitis, when the location is found within about 8 hours from the perforation event, and the perforation size is larger than $10 \mathrm{~mm}$. If the perforation site is at the mesenteric border, the surgeon will have difficulty finding the perforation. Thus, it may be helpful to carefully mark the perforation location. However, if perforation site is at the antimesenteric border, it may be visible in the operative field and it is not necessary to mark the perforation site. Simple closure or segmental resection is determined by the surgeon's experience.

Third, exploratory laparotomy may be considered when the time interval from perforation event to surgery is delayed, and with overt severe peritonitis and peritoneal contamination. However, the recovery time, scar formation, cost, and malpractice claims associated iatrogenic perforation and laparotomy will cause difficulty for endoscopists and patients.

It is important to choose appropriate management in patients with iatrogenic colon perforation. To avoid treatment failure, endoscopic clip closure, laparoscopic surgery, and exploratory laparotomy are considered according to the time interval from diagnosis, perforation size, bowel cleansing status, and evidence of overt peritonitis.

\section{Conflicts of Interest}

The author has no financial conflicts of interest.

\section{REFERENCES}

1. ASGE Standards of Practice Committee, Fisher DA, Maple JT, et al. Complications of colonoscopy. Gastrointest Endosc 2011;74:745-752.

2. Strum WB. Colorectal adenomas. N Engl J Med 2016;374:1065-1075.

3. Choi MH, Choi YS, So CS, et al. The iatrogenic complications of colonoscopic polypectomy: a multicenter retrospective study. Intest Res 2013;11:46-51.

4. Shin DK, Shin SY, Park CY, et al. Optimal methods for the management of iatrogenic colonoscopic perforation. Clin Endosc 2016;49:282-288.

5. Cha JM. Would you recommend screening colonoscopy for the very elderly? Intest Res 2014;12:275-280.

6. An SB, Shin DW, Kim JY, Park SG, Lee BH, Kim JW. Decision-making in the management of colonoscopic perforation: a multicentre retrospective study. Surg Endosc 2015 Oct 20 [Epub]. http://dx.doi. org/10.1007/s00464-015-4577-Z 